\title{
Connexin and Pannexin hemichannels are regulated by redox potential
}

\author{
Mauricio A. Retamal* \\ Facultad de Medicina Clínica Alemana, Centro de Fisiología Celular e Integrativa, Universidad del Desarrollo, Santiago, Chile
}

\section{Edited by:}

Juan C. Sáez, Pontificia Universidad

Católica de Chile, Chile

\section{Reviewed by:}

John Cuppoletti, University of

Cincinnati, USA

Jorge Arreola, Universidad

Autónoma de San Luis Potosi,

Mexico

\section{*Correspondence:}

Mauricio A. Retamal, Facultad de Medicina Clínica Alemana, Centro de Fisiología Celular e Integrativa, Universidad del Desarrollo, Avenida Las Condes \#12438, Santiago, Chile e-mail:mretamal@udd.cl
Connexins (Cxs) and Pannexins (Panxs) are two non-related protein families, having both the property to form hemichannels at the plasma membrane. There are 21 genes coding for different $\mathrm{Cx}$ based proteins and only 3 for Panx. Under physiological conditions, these hemichannels (Cxs and Panxs) present a low open probability, but when open, they allow the release of signaling molecules to the extracellular space. However, under pathological conditions, these hemichannels increase their open probability, inducing important lysis of metabolites, and ionic imbalance, which in turn induce the massive entry of $\mathrm{Ca}^{+2}$ to the cell. Actually, it is well recognized that Cxs and Panxs based channels play an important role in several diseases and -in many cases- this is associated with an aberrant hemichannel opening. Hemichannel opening and closing are controlled by a plethora of signaling including changes of the voltage plasma membrane, protein-protein interactions, and several posttranslational modifications, including protein cleavage, phosphorylation, glycosylation, hydroxylation and S-nitrosylation, among others. In particular, it has been recently shown that the cellular redox status modulates the opening/closing and permeability of at least $\mathrm{C} \times 43, \mathrm{C} \times 46$, and Panx1 hemichannels. Thus, for example, the gaseous transmitter nitric oxide (NO) can induce the S-nitrosylation of these proteins modulating in turn several of their properties. The reason is that the redox status of a cell is fundamental to set their response to the environment and also plays an important role in several pathologies. In this review, I will discuss how NO and other molecules associated with redox signaling modulate Cxs and Panx hemichannels properties.

Keywords: carbon monoxide, nitric oxide, connexin, S-Nitrosylation, redox signaling

\section{CONNEXIN AND PANNEXIN HEMICHANNELS: GENERAL PROPERTIES}

Connexins (Cxs) are a family of proteins constituted by $21 \mathrm{mem}$ bers, while Pannexins (Panxs) have only 3 members. These two protein families are formed by fourth transmembrane domains, two extracellular loops, one intracellular loop and both the N and C-terminus located at the cytoplasm (Milks et al., 1988; Yeager and Gilula, 1992; Wang and Dahl, 2010). In spite of their topological similarities, Cxs and Panx share a very low homology in terms of their amino acidic sequence. However, in both cases the oligomerization of six subunits forms a channel frequently call "hemichannel." But, it has been recently reported that Panx2 seems to form an octamer (Ambrosi et al., 2010), which would be the only exception to the hexameric hemichannels rule. Originally, the term "hemichannel" comes from the idea that the serial docking of two $\mathrm{Cx}$-hemichannels form a gap junction channel (GJC). Therefore, a half GJC should be a hemi-channel. Even though the term hemichannel is widely used, some authors also used the word connexon to refer to these channels. As mentioned above, in the case of Panx, the term hemichannel is also used, but—as they seem not to form GJCs (Scemes et al., 2007) some

Abbreviations: CO, Carbon Monoxide; NO, Nitric oxide; $\mathrm{Cx}$, Connexin; GJC, Gap junction channel; Panx, Pannexin; Cys, Cysteine. authors have suggested to call them just channels (Sosinsky et al., 2011).

Cx- based GJC and hemichannels are formed by the same protein, but they have marked differences in terms of their cellular localization, opening and closing regulation and their roles in cellular processes. Thus, Cx-hemichannels are located at the plasma membrane zone that is not contacting with other cells, whereas GJC are located at the contacting zone. In the case of Panx- hemichannels, the history is more complex. There are a number of studies that support the idea that Panxs form only hemichannels in vivo (Scemes et al., 2007), probably because Panxs are glycoproteins and its posttranslational modification could interfere with the GJC formation (Penuela et al., 2007). However, Panx1 has been observed to form GJC in Xenopus Laevis oocytes heterologous expression system (Bruzzone et al., 2003), which indicates that at least Panxl, under certain conditions, can form intercellular channels. Due to their cellular localization, when hemichannels open the flow of molecules and ions between the intracellular compartment and the extracellular space is allowed. In particular, $\mathrm{Cx}$ - hemichannels have been associated with cell-cell autocrine/paracrine communication through ATP (Romanello and D'Andrea, 2001; Stout et al., 2002), glutamate (Ye et al., 2003), cyclic ADP-ribose [cADPR] (Bruzzone et al., 2001), cAMP (Valiunas, 2013) and PGE2 (release) and glucose uptake (Retamal et al., 2007a). Additionally, hemichannels 
are relevant players in calcium waves propagation (Cotrina et al., 1998; Stout et al., 2002), memory consolidation in the amygdala (Stehberg et al., 2012), cell proliferation (Song et al., 2010), cell migration (Cotrina et al., 2008), light processing by the retina (Kamermans et al., 2001; Vroman et al., 2013), among others. On the other hand, GJC allow the cells to share ions and metabolites directly (Sáez et al., 1989; Kam et al., 1998; Goldberg et al., 1999; Niessen et al., 2000). So far, Panxl hemichannels have been shown to be permeable to ATP (Bao et al., 2004; Penuela et al., 2013) and, interestingly, it is probable to be the largest pore associated with the activation of the P2X7 receptor by extracellular ATP (Pelegrin and Surprenant, 2006; Iglesias et al., 2008). Thus, both $\mathrm{Cx}$ - and Panx- hemichannels are permeable to signaling molecules and, therefore, are associated with a great number of biological processes.

Taken together above evidence, it is now increasingly accepted that under physiological conditions Cxs- hemichannels can open, but with a low open probability (Contreras et al., 2003), which would be enough to participate in several cellular processes (Sáez et al., 2010; Rackauskas et al., 2010; Kar et al., 2012). However, under pathological conditions, $\mathrm{Cx}$ - hemichannels increase their overall activity most likely due to increasing the open probability and thus forming "leaky hemichannels" (Liang et al., 2005; Stong et al., 2006; Sánchez et al., 2010) and/or increasing their number at the plasma membrane (Retamal et al., 2006). This augmented hemichannel activity has been associated with an accelerated cell death in heterologous systems (Essenfelder et al., 2004; Gerido et al., 2007; Tong et al., 2011; Levit et al., 2012), supporting the idea that a low hemichannel activity can be related to several cell functions, but a high and/or uncontrolled hemichannel activity diminishes cell viability. Similarly, Panx- hemichannels also increase their activity under pathological conditions, thus Panx1 hemichannels increase their opening probability in cells metabolically inhibited (Domercq et al., 2010; Bargiotas et al., 2011), as well as under inflammatory conditions (Riteau et al., 2010; Orellana et al., 2011).

As presented before, maintaining a controlled opening/closing hemichannel is very important to preserve a normal cell function. Cx hemichannels are constantly under the control of several factors, including those acting intracellularly, as membrane potential (Ebihara, 2003; Bukauskas and Verselis, 2004; Kronengold et al., 2012), intramolecular interactions (Ponsaerts et al., 2010), pH (Peracchia, 2004) and posttranslational modifications, such as phosphorylation (Sáez et al., 1998; Lampe and Lau, 2000; Moreno, 2005), ubiquitination, SUMOylation, palmitoylation, caspasecleavage, S-Nitrosylation, hydroxylation and deamidation (reviewed by Johnstone et al., 2012; D'Hondt et al., 2013), as well as those acting extracellularly, such as $\mathrm{Ca}^{2+}$ and $\mathrm{Mg}^{2+}$ (Verselis and Srinivas, 2008; Bader et al., 2012). Similarly, Panx- hemichannels are also modulated by intracellular signaling molecules and posttranslational modifications, such as N-glycosylation in their extracellular loops [Panx1, Panx2, and Panx3, asparagine 254, 86, and 71, respectively] (Penuela et al., 2013). Notwithstanding, there is no confirmation yet that Panx are phosphoproteins; current evidence indicates that they can be so. Thus, a connection was observed between activation of kinases, such as Src (Weilinger et al., 2012) and Rho (Seminario-Vidal et al., 2009) and the Panxl hemichannel opening. Finally, rises in the intracellular calcium concentration do increase the Panx1 hemichannel opening (Locovei et al., 2006). In conclusion, under physiological conditions, these molecular mechanisms act in combination to keep the hemichannels mostly closed. This mini review will focus on the effect of reactive oxygen species [ROS, i.e., nitric oxide (NO)] over the permeability and gating of Cxs and Panxs hemichannels.

\section{CONNEXIN'S HEMICHANNELS AND THEIR CONTROL BY REDOX SIGNALING MOLECULES}

Probably the first study suggesting that Cxs- hemichannels can be modulated by redox signaling molecules was the work performed by Contreras and his co-workers (2002). This work showed that rat's astrocytes in culture become permeable to fluorescent tracers Ethidium and Lucifer yellow, after being metabolically inhibited by $75 \mathrm{~min}$ with iodoacetic acid and antimycin A. This membrane permeabilization was blocked by Octanol, $\mathrm{La}^{3+}$ and $18 \alpha$-glycyrrhetinic acid (AGA), three well known hemichannel blockers, and was not observed in astrocytes from mouse knockout for $\mathrm{Cx} 43$. These results indicate that metabolic inhibition induces the opening of $\mathrm{Cx} 43$ hemichannels. Additionally, it was observed that Trolox -a free radical scavenger- was very efficient in preventing the opening of hemichannels induced by metabolic inhibition (Contreras et al., 2002). This indicates that metabolic stress increases free radicals concentration by an unknown mechanism (at least in that date) inducing the Cx43 hemichannel opening. Interestingly, a progressive dephosphorylation of this protein was also observed in parallel with the metabolic inhibition progression. But even when $\mathrm{Cx} 43$ is dephosphorylated, which is a signaling to induce hemichannel opening (Bao et al., 2007), hemichannels were closed by Trolox indicating that the redox status of $\mathrm{Cx} 43$ is a control mechanism more important than dephosphorylation, at least in metabolic stress conditions. Then, Retamal et al. (2006) studied the molecular mechanism that control Cx43 hemichannel opening under metabolic inhibition. Here, it was observed that astrocytes under control conditions were permeabilized to Ethidium after being exposed to a nitric oxide (NO) donor (GSNO). This permeabilization was reverted by DTT, indicating that NO induces the opening of $\mathrm{Cx} 43$ hemichannels by a mechanism dependent of S-nitrosylation (Retamal et al., 2006). Because of the above, the effect of DTT was tested in astrocytes under metabolic inhibition, and it was observed that DTT was able to block the entry of Ethidium into the cells. A similar result was observed when a reduced gluthation that is able to cross the plasma membrane (GSH-EE) was added to the bath solution, but this reduction was not observed when GSH (which does not cross the membrane) was added (Retamal et al., 2006). This indicates that the hemichannel blocking induced by reducing agents is due to reduction of the oxidized intracellular cysteines (Cys). At this point, it was not yet determined which $\mathrm{Cx} 43$ Cys were modified by NO, but it was demonstrated that $\mathrm{Cx} 43$ is S-nitrosylated by GSNO and also by metabolic inhibition, thus changing some properties of these channels.

Then, we studied the effect of redox molecules in $\mathrm{Cx} 43$ expressed in HeLa cells (Retamal et al., 2007b). First, we studied the effect of GSNO on these hemichannels, and unexpectedly 
no changes were observed in the activity of $\mathrm{Cx} 43$ hemichannels (data not published). However, it was evident that a reducing agent such as DTT presents a robust activation of these hemichannels, which was observed as an increase of dye uptake and by electrophysiology experiments. This indicates that HeLa cells somehow keep Cx43 hemichannel's Cys groups oxidized and, therefore, susceptible to be reduced by reducing agents such as dithiothreitol (DTT). Now, in astrocytes, oxidative stress open hemichannels and in HeLa cells reducing agents induce the opening of hemichannels. How can this be possible? To answer this question, Retamal et al. (2007b) added DTT to cells under metabolic inhibition at different times and it was observed that, when DTT was added before 20 min of metabolic inhibition, it induced an increase in Ethidium uptake. Moreover, when DTT was added around $30 \mathrm{~min}$ of metabolic inhibition no clear effect was observed, but when added after $40 \mathrm{~min}$, it induced closure of Cx43 hemichannels. These data suggest that even when Cx43 hemichannels are affected by the cellular redox potential, the net effect is probably going to depend upon some other factors, such as the phosphorylation/dephosphorylation balance. Thus, it is necessary to elucidate the possible cross-talk between phosphorylation, $\mathrm{pH}$, and membrane potential within the final effect induced by oxidation. Experiments based on this line of thought have been published (De Vuyst et al., 2009), but more research is still needed.

As mentioned before, $\mathrm{Cx} 43$ hemichannels are sensitive to redox potential, but until now there is no evidence showing which is or are the Cys groups that can be modulated by NO or other free radicals. It has been suggested that Cys271 could be a good candidate, because this Cys has been shown to be Snitrosylated in GJC formed by $\mathrm{Cx} 43$ in endothelial cells (Straub et al., 2011). Interestingly, this posttranslational modification seems to decrease the permeability to $\mathrm{IP}_{3}$ through $\mathrm{Cx} 43$ GJC, and it is constantly modulated by the S-nitrosogluthathion reductase (Straub et al., 2011).

In another experiment performed in astrocytes in culture, the effect of proinflammatory cytokines on GJC and hemichannels were analyzed. Under these conditions, GJC between astrocytes were found to be closed, measured as a decrease in the intercellular transference of Lucifer yellow, but in parallel it was observed an increase in Ethidium uptake, which would suggest the opening of Cx43 hemichannels (Retamal et al., 2007a). The increase of hemichannel activity was prevented by L-name (an inhibitor of the nitric oxide syntase) and also by DTT. But, the decrease of cell coupling was affected neither by DTT nor by L-name (Retamal et al., 2007a). Above findings suggest that Cx43 hemichannels are more sensitive to changes in the redox potential than GJC and/or the modifications in different Cys groups show differences in terms of sensitivity to reducing agents. Similar results have been observed in ryanodine receptors, where different Cys groups are differentially oxidized (i.e., by $S$-nitrosylation, $S$ glutathionylation, and disulfide oxidation) and induced different modification to channel properties (Aracena-Parks et al., 2006). On the other hand, the increase of $\mathrm{Cx} 43$ hemichannels activity in astrocytes induced by NO can lead to a massive neuronal death due to considerable efflux of glutamate from astrocytes (Froger et al., 2010) and the inhibition of Cx43 hemichannels by synthetic cannabinoids was neuroprotective (Froger et al., 2010). Therefore, more studies are necessary to find out the effect of other oxidizing agents that affect Cys groups, such as oxidized glutathione, which will help to understand the role of redox potential as controller of hemichannels in pathological and also physiological conditions.

It has been recently reported that $\mathrm{Cx} 46$ is also modulated by the NO donor GSNO (Retamal et al., 2009). In this work, GSNO was shown to induce changes in $\mathrm{Cx} 46$ hemichannel opening and closing kinetics, current-voltage relationship and also induces the appearance of a current relaxation at voltages over $+40 \mathrm{mV}$. All of them are reverted by DTT, suggesting that GSNO can induce the oxidation of $\mathrm{Cx} 46$ in Cys groups. These modifications were not observed in a $\mathrm{Cx} 46$ without intracellular Cys (Cx46C3A), thus suggesting that some of the two intracellular Cys are S-nitrosylated. Additionally, it was observed that GSNO induces a slight (but statistically significant) decrease in the hemichannel permeability to large molecules (i.e., Lucifer yellow) (Retamal et al., 2009). To date, it has not been possible to elucidate which is/are the Cys involved in this phenomenon. However, these data seem to be relevant to understand the role of free radicals as initiators and/or enhancers of lens opacity (disease commonly known as cataracts) (Berthoud and Beyer, 2009; Retamal et al., 2011).

A recent study has shown that $\mathrm{Cx} 32, \mathrm{Cx} 37$, and $\mathrm{Cx} 40$ are also affected by NO (Figueroa et al., 2013). In this work, it was determined that NO induced the opening of $\mathrm{Cx} 37$ and $\mathrm{Cx} 40$, whereas it induced the closure of $\mathrm{Cx} 32$. In all cases (including Cx43), hemichannels were permeable to NO (Figueroa et al., 2013). This work suggests that hemichannels formed by $\mathrm{Cx} 32,37,40$, and 43 could be good pathways for the diffusion of NO between endothelial cells and smooth muscle cells.

Finally, hemichannel opening has been observed in other models of oxidative stress, such as cadmium-induced

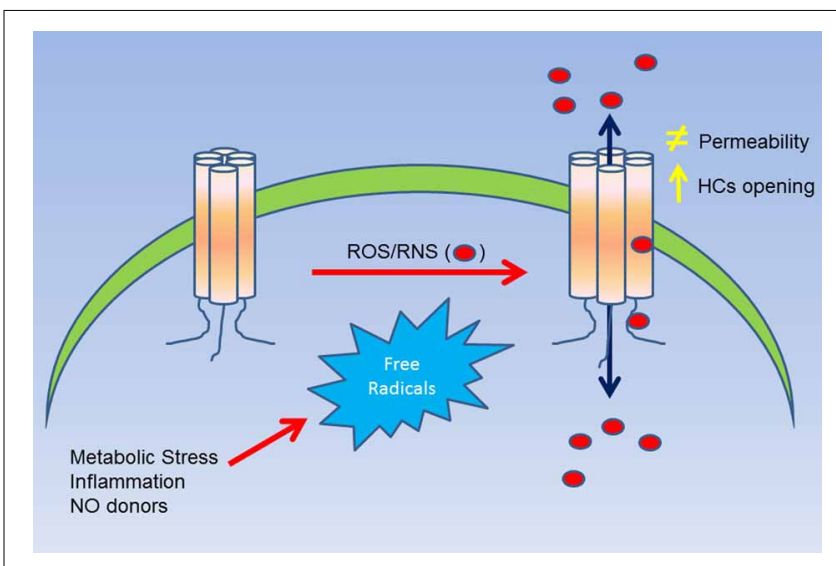

FIGURE 1 | Summary of the effect of ROS/RNS over Cxs hemichannels. A cellular stress (i.e., metabolic inhibition) induces an increase of ROS/RNS production, which in turn, can affect directly $\mathrm{Cx}$ hemichannels (as observed in Cys271 of $\mathrm{Cx} 43$ ). This molecular modification can induce and increase in the open probability of hemichannels and/or changes in their permeability to large molecules. Additionally, the posttranslational modification induced by ROS/RNS can also lead to an increase of the permeability of nitro oxide and possibly of other free radicals as well. 
Table 1 | List of all Cxs and Panx hemichannels that are known to be affected by changes of cellular redox potential.

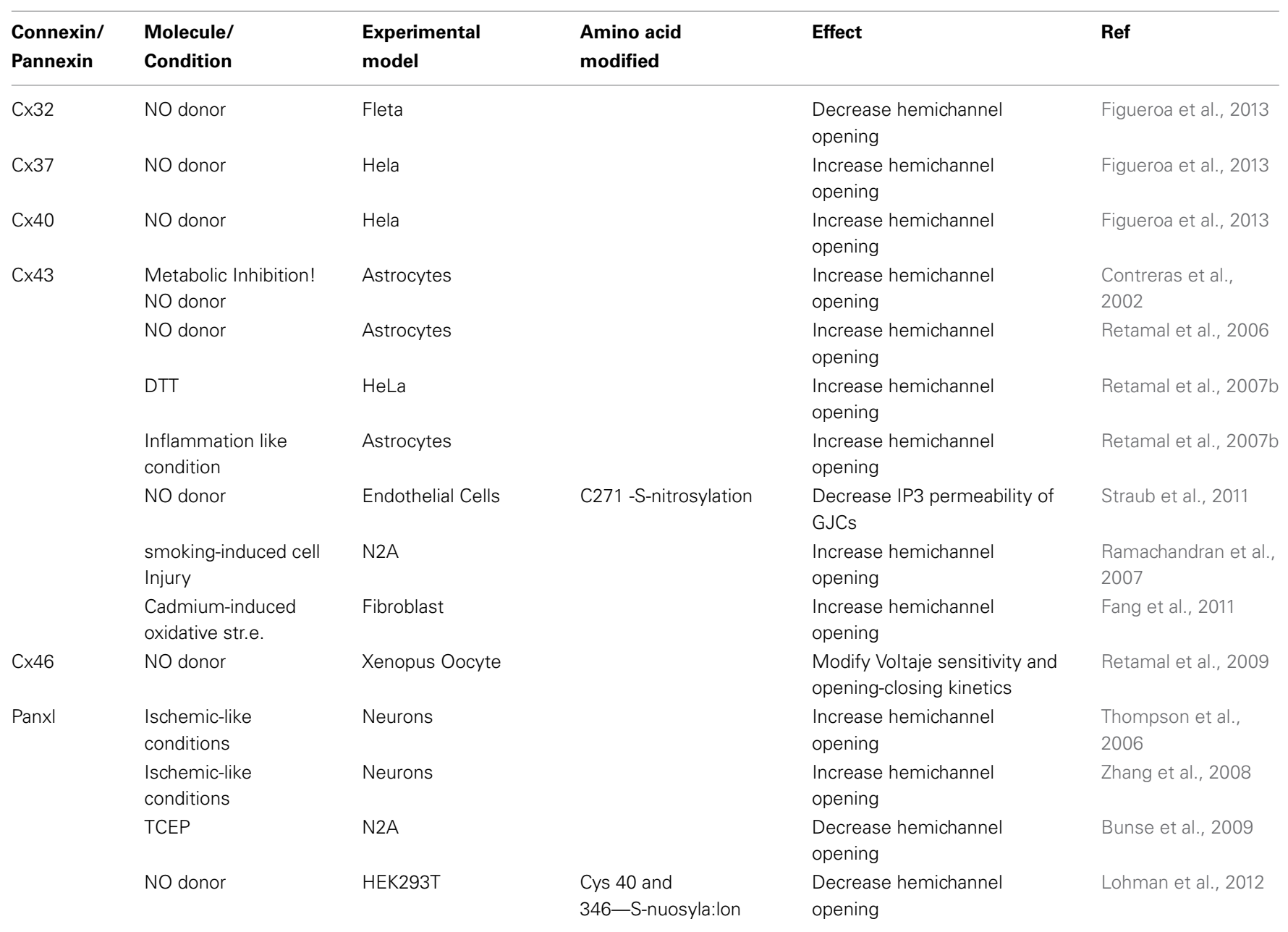

This table specifies the model in which the experiments were performed, the observed effects on hemichannel activity and which Cys group was modified.

oxidative stress (Fang et al., 2011) and smoking-induced cell injury (Ramachandran et al., 2007). In the work of ramachandran, they observed that CSE (cigarette smoke extract) and $\mathrm{h}_{202}$ were able to cross the plasma membrane through the open hemichannels. This is consistent with the work of Figueroa et al. (2013), suggesting that hemichannels are not only affected by free radicals but also are permeable to them (Figure 1). Interestingly, in 2012 a mutant of $\mathrm{Cx} 31$ (Cx31R42P) was reported to produce hemichannels with a gain in activity, which induced cell death (Chi et al., 2012). This cell death was prevented by hemichannel blockers, increasing the extracellular concentration of $\mathrm{Ca}^{2+}$, and by the ROS scavenger butylated hydroxyanisole (BHA) (Chi et al., 2012). In this work, authors suggested that this mutation somehow would induce an increase in the ROS production by the cells. Moreover, as $\mathrm{Cx} 31$ has several cys groups in their c-terminus, it is possible that these ROS are oxidizing Cx31 hemichannels, which in turn would induce the hemichannel opening, similarly to previous observation made in $\mathrm{Cx} 43$ hemichannels expressing cells (Contreras et al., 2002; Retamal et al., 2006).

To date, no direct evidence is available to support the possibility that gsgg, $\mathrm{h}_{202}$, carbon monoxide (co), hydrogen sulphide $\left(\mathrm{h}_{2 s}\right)$ or any other oxidant molecules may affect the properties of cx hemichannels. Therefore, it is absolutely essential to investigate the effect of these molecules in hemichannels formed by cxs in vitro and in vivo in order to better understand the role of ROS in hemichannel function.

\section{PANNEXIN'S HEMICHANNELS AND THEIR CONTROL BY REDOX SIGNALING MOLECULES}

Panx1 is expressed in several types of cells (Bruzzone et al., 2003). As observed for Cx43 hemichanels in astrocytes, neurons under ischemic-like conditions open their Panx1 hemichannels (Thompson et al., 2006), phenomenon that was inhibited by DTT and L-NAME, and suggesting that NO is involved in neuronal Panx1 hemichannel opening (Zhang et al., 2008). In the same line of evidence, Panx1 hemichannels are closed by reducing agents (Bunse et al., 2009). Accordingly, mutation of Cys 40 and 346 prevent the GSNO induced S-nitrosylation of this protein and also prevent Panx1 hemichannels opening (Lohman et al., 2012). The substitution of Cys40 or Cys346 by Serine induced the appearance of hemichannels constitutively open (Bunse et al., 2009, 2010). In contrast, mutation of Zebra fish Cys282 to tryptophan (C282W) 


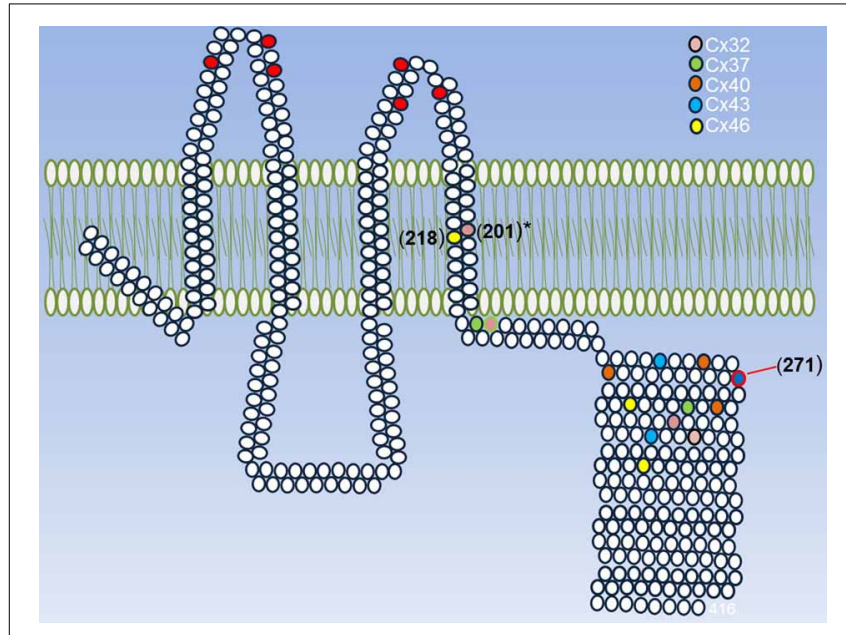

FIGURE 2 | Summary of Cys that can be modified by ROS/RNS. A representative $\mathrm{Cx}$ is shown and its conserved extracellular Cys is highlighted (red circles) and each cysteine present in Cxs 32, 37, 40, 43, and 46 are shown in different colors. These Cxs were chosen because they are sensitive to redox potential. The exact position for each Cys was taken according to their last aa in the TM4. The asterisk in Cys201 of Cx32 indicates that their mutations induce the appearance of a disease and the Cys 271 of $\mathrm{Cx} 43$ is the only one that has proved to be S-nitrosylated.

reduced the hemichannel activity (Prochnow et al., 2009). Above results suggest that these Cys groups are probably the redox potential sensor in this protein, and they can vary depending on the species. Interestingly, it has been recently proposed that Panxl hemichannels can help NO to cross the plasma membrane (Campanucci et al., 2012), phenomenon recently shown for Cx-hemichannels (Figueroa et al., 2013). Taking into account all the evidence, it is possible to suggest that under physiological conditions, intracellular reduced glutathione (GSH) keeps Panx1 hemichannel closed, but when the free radical concentration increases, due to (for example) metabolic stress, Panx1 hemichannels become open. In summary, Panx1 is affected by ROS, but the exact molecular mechanism is not completely understood yet. At present, there are no data showing the effects of ROS upon the activity/properties of hemichannels formed by Panx2 and Panx3.

\section{REDOX SIGNALING MEDIATES HEMICHANNELS FUNCTION IN PATHOLOGICAL CONDITIONS}

The role of Cxs and Panx hemichannels on pathological conditions has been extensively reviewed (Bennett et al., 2012; Orellana et al., 2013). Therefore, this section will be focused on the role of redox signaling as intermediary of cellular response mediated by Cxs and Panx hemichannels in unhealthy cells. It is well accepted that under pathological conditions there is an increase in ROS/RNS production, which can affect different proteins through posttranslational modifications (Kolluru et al., 2013; Nakamura et al., 2013), thus, affecting diverse cellular functions (Gaston et al., 2003). In this context, an enhanced production of for example NO will lead to an increased hemichannel opening, which in turn will have several consequences in the cellular function as discussed before. Now, it is important to mention that the net effect of NO on hemichannels is going to depend on the level of
NO produced. Thus, moderated levels of NO will induce a moderate hemichannel opening, which will have a profound effect in the autocrine/paracrine communication. This is because it is known that $\mathrm{Cx} 43$ hemichannel opening allows the release of signaling molecules to the extracellular space such as ATP (Stout et al., 2002), which is a well-recognized molecule involved in inflammatory processes (Eltzschig et al., 2012), mainly through P2X7 activation (Arulkumaran et al., 2011). Thus, hemichannel opening induced by NO could be a key point in several inflammatory processes. On the other hand, a large NO production may induce a massive hemichannel opening that is a signal that enhances and/or accelerates cell death (Retamal et al., 2006; Sáez et al., 2010). Therefore, depending on the NO production, the hemichannel activity may lead to a wide range of responses, from tissue inflammation to massive cell death. Table 1 summarizes the effect of ROS/RNS over Cx and Panx hemichannels.

Another important issue about redox control of hemichannel activity in physiological and pathological conditions is that at least Cxs that seem to be sensitive to NO. As connexins, ryanodine receptors show several Cys groups and have been shown that they can be differentially modify by NO (S-nitrosylation) or GSSG (Sgluthationylation) (Aracena-Parks et al., 2006). These differences in Cys modifications depend on their microenvironment in which a given Cys is located (Lam et al., 2010). Similarly, it can be postulated that different Cys in different regions on a given Cxs or Panx, could be affected by NO or GSSG in a different way and that may induce different changes in the hemichannels properties. Therefore, it is very important to study the effect of other oxidant molecules, such as $\mathrm{H}_{2} \mathrm{~S}, \mathrm{CO}, \mathrm{GSSG}$ on hemichannel activity and test which are the Cys that are modified by these molecules. Also, it would be interesting to study if these modifications are present in proteins expressed in cells under pathological conditions. Following this line of evidence, our research group found that in rat's lenses that present cataract, $\mathrm{Cx} 46$ is S-nitrosylated, which suggests that at least this Cxs is modified by NO in pathological conditions, hence validating the data obtained in vitro. In Figure 2, it is observed all Cys groups present in Cxs sensitive to redox potential that are located in the TM4 and mostly in a region between 20 and 70 aa far from the beginning of the Cterminus. Although there are no conserved Cys, it is possible to postulate a zone sensitive to ROS/RNS. Actually, a Cys 271 of Cx43 (which is already S-nitrosylated) is found in this putative zone. Cys in TM4 could be important redox sensors because $\mathrm{rCx} 46$ truncated C-terminus ( $\mathrm{Cx} 46 \Delta \mathrm{CT}$, which lacks Cys group in their C-terminus) and hCx46WT (which has only a Cys in TM4) are sensitive to nitric oxide (personal observation). Cys in a similar position-Cys201 (Morlé et al., 2000) and their homologous in Cx26-Cys202 (Sillén et al., 1998) -when mutated- induce the appearance of X-linked Charcot-Marie-Tooth disease and deafness, respectively. It is important to emphasize that there are Cxs with differences between their expression of Cys in their C-terminus (Table 2, Cx40 and Cx46), but there are others Cxs presenting important homologies (Table 2, Cx32 and Cx37) (Table 2).

NO is a highly reactive gas that participates in several physiological and pathological processes (Wang et al., 2010; Anand and Stamler, 2012; Nakamura et al., 2013). Recently, this 
Table 2 | Alignment of C-terminus of human, rat and mouse Cxs and Panx modified by nitric oxide.

\section{$\mathrm{Cx} 32$}

H-evvyliiracarragrrsnppsrkgsgfghrlspeykqneinkllseqdgslkdilrrspgtgaglaeksdrcsac

R-evvyliiracarraqrisnppsrkgsgfghrlspeykqneinkllseqdgslkdilrrspgtgaglaeksdrcsac

M-evvyliiracarraqresnppsrkgsgfghrlspeykqneinkllseqdgslkdilrrspgtgaglaeksdrcsac

Cx37

H-vhllcrclsrgmrarqgqdapptqgtssdpytdqvffylpvgqgpssppcptynglssseqnwanltteerlassrpplfldpppqngqkppsrpsssaskkqyv

R-vhllcrcvsreikarrdhdtrpaqgsasdpypeqvffylpmgegpssppcptynglssteqnwanltteerltstrpppfvnaapqggqkssspnssaskkqyv

M-vhlicrcvsreikarrdhdarpaqgsasdpypeqvffylpmgegpssppptynglssteqnwanltteerltssrpppfvntapqggrkspsrpnssaskkqyv

$\mathrm{Cx} 40$

H-yhlgwkkirqrfvkprqhmakcqlsgpsvgivqsctpppdfnqclengpggkffnpfsnnmasqqntdnlvteqvrgqeqtpgegfiqvrygqkpevpngvspghrlphgyhsdkrrlskasskarsddlsc R-yhlgwkkirqrlaksrqg-dkhql lgpstslvqgltpppdfnqclknspdekffsdfsnnmgsrknpdplateevpnqeqipeegfihtqygqkpeqpsgasaghrfpqgyhsdkrrlskasskarsddlsc M-yhlgwkkirqrfgksrqgvdkhqlpgpptslvqsltpppdfnqclknssgekffsdfsnnmgsrknpdalatgevpnqeqipgegfihmhysqkpeyasgasaghrlpqgyhsdkrrlskasskarsddlsv

$\mathrm{Cx} 43$

H-elfyvffkgvkdrvkgksdpyhatsgalspakdcgsqkyay fngcssptaplspmsppgyklvtgdrnnsscrnynkqaseqnwanysaeqnrmgqagstisnshaqpfdfpddnqnskklaaghelqplaiv R-elfyvffkgvkdrvkgrsdpyhattgplspskdcgspkyayfngcssptaplspmsppgyklvtgdrnnsscrnynkqaseqnwanysaeqn rmgqagstisnshaqpfdfpddnqnakkvaaghelqplaiv M-elfyvffkgvkdrvkgrsdpyhattgplspskdcgspkyayfngcssptaplspmsppgyklvtgdrnnsscrnynkqaseqnwanysaeqnrmgqagstisnshaqpfdfpddsqnakkvaaghelqplaiv

dqrpssrassrassrprpddlei

dqrpssrassrassrprpddlei

dqrpssrassrassrprpddlei

Cx46

H-ei yhlgwkklkqgvtsrlgpdaseaplgtadppplppssrppavaigfppyyahtaaplgqaravgypgapppaadfkllalteargkgqsaklynghhhllmteqnwanqaaerqppalkaypaastpaaps R-eiyhlgwkklkqgvtnhfnpdasevrhkpldplseaansgppsvsiglppyythpacptvqgkatgfpgapllpadftvvtlndaqgrghpvkhcnghh--ltteqnwaslgaepqtpaskpssaas sp---M--iyhlgwkklkqgvtnh fnpdasearhkpldplptatssgppsvsigfppyythpacptvqakaigfpgapl spadftvvtlndaqgrnhpvkhcnghh--ltteqnwtrqvaeqqtpaskpssaassp----

pvgssspplaheaeagaapllldgsqsslegsalagtpeeeqavttaaqmhqpplplgdpgraskasrassgrarpedlaj

------hgrkg----ltdssgssleesalvvtp-egeqalattvemhspplvlldperssk---sssgrarpgdlai -dgrkg----lidssgsslgesalvvtpeegeqalattvemhspplvildpgrssk---ssngrarpgdlai

\section{$\operatorname{Panx} 1$}

H-tlfvpfrqktdvlkvyeilptfdvlhfksegyndlslynlfleenisevksykclkvlenikssgqgidpmllltnlgmikmdvvdgktpmsaemr-eeqgnqtaelqgmnidsetkanngeknarqrllds R-tlfvpfrqktdvl kvyeilptfdvlhfksegyndlslynlfleeniselksykclkvleniksngqgidpmllitnlgmikmdvidgkvpmslqtkgedqgsqrmdfkdldlssetaanngeknsrqrl lns M-tffipfrqktdilkvyeilptfdvlhfksegyndlslynlfleeniselksykclkvleniksngqgidpmllltnlgmikmdiidgkiptslqtkgedqgsqrvefkdldlsseaaanngeknsrqrllnp

$\mathrm{SC}$

Cys in each C-terminus protein is shown in yellow. Note that all Cys are conserved in Cx32 in spite of large differences in Cx46. Human Cx46 has no Cys in C-terminus, but a Cys is present in position 218, in transmembrane 4. It has been reported that Cys located in the membrane can be S-nitrosylated (Leclerc et al., 2006). Therefore, the possibility that $h C x 46$ can be redox sensitive cannot be ruled out.

gaseous transmitter has been shown to be permeable through hemichannels formed by Cxs (Figueroa et al., 2013). Considering that Cxs and Panx hemichannels are modulated by NO (Retamal et al., 2006, 2009; Lohman et al., 2012), it is possible to suggest that under pathological conditions hemichannels are playing an amplifier role of signaling pathways activated by $\mathrm{NO}$, and -additionally- allowing NO to diffuse easily and widely in a tissue. On the other hand, it is known that there is a good correlation between intracellular $\mathrm{Ca}^{2+}$ levels and NO production. Recently, it has been demonstrated that $\mathrm{Cx} 26$ and $\mathrm{Cx} 43$ hemichannels are permeable to this cationic divalent ion (Sánchez et al., 2010; Schalper et al., 2010; Fiori et al., 2012). Thus, under pathological conditions where hemichannels increase their open probability, hemichannels can induce intracellular $\mathrm{Ca}^{2+}$ increase, which in turn can activate intracellular pathways, such as caspases (Ishiura, 1981) and NO production (Schmidt et al., 1992). In this context, it has been recently shown that hemichannels participate in the NO production in injured endothelial cells of aorta (Berra-Romani et al., 2013). Therefore, several cellular responses induced by redox molecules (oxidant or reducing ones), such as; increased intercellular communication mediated by ATP and activation of $\mathrm{Ca}^{2+}$ dependent intracellular signaling pathways, can be mediated, at least in part, by inducing hemichannel opening. Finally, since the intracellular $\mathrm{Ca}^{2+}$ concentration is a good indicator of cellular health (Rasmussen et al., 1990), hemichannel opening under pathological conditions can set not only the cell response to an injury, but also determine the final destination of a cell.

\section{CONCLUSIONS AND PERSPECTIVES}

For many years, much research has been performed to elucidate the molecular mechanism involved in the control of opening and closing of $\mathrm{Cx}$ and Panx- hemichannels, while the exact molecular mechanism has not yet been resolved. The most studied are probably: changes of membrane potential, phosphorylation, $\mathrm{pH}$ and extracellular divalent cations. Recently, another control mechanism has been proposed and it seems to affect several different types of Cxs and- at least- Panxl hemichannel function. This new control mechanism is linked to changes of redox potential. Presently, the effect of NO upon $\mathrm{Cx}$ - and Panx hemichannels has been the most studied. However, there are other important free radicals that act in both pathological and physiological conditions. I would like to point out that carbon monoxide $(\mathrm{CO})$ and hydrogen sulphide $\left(\mathrm{H}_{2} \mathrm{~S}\right)$ are emerging as important gaseous transmitters with relevant roles in cell physiology (Wilkinson and Kemp, 2011; Ju et al., 2013). Thus, future lines of research will be probably focused on: (1) the effect of $\mathrm{CO}$ and $\mathrm{H}_{2} \mathrm{~S}$ upon Cxs and Panx- hemichannels and GJCs; (2) Which is or are-at molecular level- the redox sensor(s) in different Cxs and Panxs, (3) Which are (if any) the hypothetical interactions between the redox sensor and the wellrecognized slow and fast gating of Cxs hemichannels, and (4) 
which are the modifications on the structure of the Cxs in terms of folding, protein-protein bindings and intramolecular interaction.

\section{ACKNOWLEDGMENTS}

I would like to thank Mrs Carolina Larrain for her help in preparing this manuscript. This work was funded by grants Fondecyt \#1120214 and Anillo \#ACT1104.

\section{REFERENCES}

Ambrosi, C., Gassmann, O., Pranskevich, J. N., Boassa, D., Smock, A., Wang, J., et al. (2010). Pannexin1 and Pannexin2 channels show quaternary similarities to connexons and different oligomerization numbers from each other. J. Biol. Chem. 285, 24420-24431. doi: 10.1074/jbc.M110.115444

Anand, P., and Stamler, J. S. (2012). Enzymatic mechanisms regulating protein S-nitrosylation: implications in health and disease. J. Mol. Med. (Berl). 90, 233-244. doi: 10.1007/s00109-012-0878-z

Aracena-Parks, P., Goonasekera, S. A., Gilman, C. P., Dirksen, R. T., Hidalgo, C., and Hamilton, S. L. (2006). Identification of cysteines involved in Snitrosylation, S-glutathionylation, and oxidation to disulfides inryanodine receptor type 1. J. Biol. Chem. 281, 40354-40368. doi: 10.1074/jbc.M600876200

Arulkumaran, N., Unwin, R. J., and Tam, F. W. (2011). A potential therapeutic role for $\mathrm{P} 2 \mathrm{X} 7$ receptor (P2X7R) antagonists in the treatment of inflammatory diseases. Expert Opin. Investig. Drugs 20, 897-915. doi: $10.1517 / 13543784.2011 .578068$

Bader, P., Weingart, R., and Egger, M. (2012). Regulation of Cx45 hemichannels mediated by extracellular and intracellular calcium. Pflugers Arch. 464, 249-259. doi: 10.1007/s00424-012-1133-8

Bao, L., Locovei, S., and Dahl, G. (2004). Pannexin membrane channels are mechanosensitive conduits for ATP. FEBS Lett. 572, 65-68. doi: 10.1016/j.febslet.2004.07.009

Bao, X., Lee, S. C., Reuss, L., and Altenberg, G. A. (2007). Change in permeant size selectivity by phosphorylation of connexin 43 gap-junctional hemichannels by PKC. Proc. Natl. Acad. Sci. U.S.A. 104, 4919-4924. doi: 10.1073/pnas.0603154104

Bargiotas, P., Krenz, A., Hormuzdi, S. G., Ridder, D. A., Herb, A., Barakat, W., et al. (2011). Pannexins in ischemia-induced neurodegeneration. Proc. Natl. Acad. Sci. U.S.A. 108, 20772-20777. doi: 10.1073 /PNAS.1018262108

Bennett, M. V., Garré, J. M., Orellana, J. A., Bukauskas, F. F., Nedergaard, M., and Sáez, J. C. (2012). Connexin and pannexin hemichannels in inflammatory responses of glia and neurons. Brain Res. 1487, 3-15. doi: 10.1016/j.brainres.2012.08.042

Berra-Romani, R., Avelino-Cruz, J. E., Raqeeb, A., Della Corte, A., Cinelli, M., Montagnani, S., et al. (2013). $\mathrm{Ca}^{2+}$ dependent nitric oxide release in the injured endothelium of excised rat aorta: a promising mechanism applying in vascular prosthetic devices in aging patients. BMC Surg. 13(Suppl. 2), S40. doi: 10.1186/1471-2482-13-S2-S40

Berthoud, V. M., and Beyer, E. C. (2009). Oxidative stress, lens gap junctions, and cataracts. Antioxid. Redox Signal. 11, 339-353. doi: 10.1089/ars.2008.2119

Bukauskas, F. F., and Verselis, V. K. (2004). Gap junction channel gating. Biochim. Biophys. Acta. 1662, 42-60. doi: 10.1016/j.bbamem.2004.01.008

Bruzzone, S., Guida, L., Zocchi, E., Franco, L., and De Flora, A. (2001). Connexin 43 hemichannels mediate $\mathrm{Ca}^{2+}$-regulated transmembrane NAD+ fluxes in intact cells. FASEB J. 15, 10-12. doi: 10.1096/fj.00-0566fje

Bruzzone, R., Hormuzdi, S. G., Barbe, M. T., Herb, A., and Monyer, H. (2003). Pannexins, a family of gap junction proteins expressed in brain. Proc. Natl. Acad. Sci. U.S.A. 100, 13644-13649. doi: 10.1073/pnas.2233464100

Bunse, S., Locovei, S., Schmidt, M., Qiu, F., Zoidl, G., Dahl, G., et al. (2009). The potassium channel subunit Kvbeta3 interacts with pannexin 1 and attenuates its sensitivity to changes in redox potentials. FEBS J. 276, 6258-6270. doi: 10.1111/j.1742-4658.2009.07334.x

Bunse, S., Schmidt, M., Prochnow, N., Zoidl, G., and Dermietzel, R. (2010). Intracellular cysteine 346 is essentially involved in regulating Panxl channel activity. J. Biol. Chem. 285, 38444-38452. doi: 10.1074/jbc.M110.101014

Campanucci, V. A., Dookhoo, L., Vollmer, C., and Nurse, C. A. (2012). Modulation of the carotid body sensory discharge by NO: an up-dated hypothesis. Respir. Physiol. Neurobiol. 184, 149-157. doi: 10.1016/j.resp.2012.04.005
Chi, J., Li, L., Liu, M., Tan, J., Tang, C., Pan, Q., et al. (2012). Pathogenic connexin31 forms constitutively active hemichannels to promote necrotic cell death. PLoS ONE. 7:e32531. doi: 10.1371/journal.pone.0032531

Contreras, J. E., Sánchez, H. A., Eugenin, E. A., Speidel, D., Theis, M., Willecke, K., et al. (2002). Metabolic inhibition induces opening of unapposed connexin 43 gap junction hemichannels and reduces gap junctional communication in cortical astrocytes in culture. Proc. Natl. Acad. Sci. U.S.A. 99, 495-500. doi: 10.1073/pnas.012589799

Contreras, J. E., Sáez, J. C., Bukauskas, F. F., and Bennett, M. V. (2003). Gating and regulation of connexin $43(\mathrm{Cx} 43)$ hemichannels. Proc. Natl. Acad. Sci. U.S.A. 100, 11388-11393. doi: 10.1073/pnas.1434298100

Cotrina, M. L., Lin, J. H., Alves-Rodrigues, A., Liu, S., Li, J., Azmi-Ghadimi, H., et al. (1998). Connexins regulate calcium signaling by controlling ATP release. Proc. Natl. Acad. Sci. U.S.A. 95, 15735-15740. doi: 10.1073/pnas.95.26.15735

Cotrina, M. L., Lin, J. H., and Nedergaard, M. (2008). Adhesive properties of connexin hemichannels. Glia 56, 1791-1798. doi: 10.1002/glia.20728

D’Hondt, C., Iyyathurai, J., Vinken, M., Rogiers, V., Leybaert, L., Himpens, B., et al. (2013). Regulation of connexin-and pannexin-based channels by post-translational modifications. Biol. Cell 105, 373-398. doi: 10.1111/boc.201200096

De Vuyst, E., Wang, N., Decrock, E., De Bock, M., Vinken, M., Van Moorhem, M., et al. (2009). $\mathrm{Ca}(2+)$ regulation of connexin 43 hemichannels in $\mathrm{C} 6$ glioma and glial cells.Cell Calcium 46, 176-187. doi: 10.1016/j.ceca.2009.07.002

Domercq, M., Perez-Samartin, A., Aparicio, D., Alberdi, E., Pampliega, O., and Matute, C. (2010). P2X7 receptors mediate ischemic damage to oligodendrocytes. Glia 58, 730-740. doi: 10.1002/glia.20958

Ebihara, L. (2003). Physiology and biophysics of hemi-gap-junctional channels expressed in Xenopus oocytes. Acta Physiol. Scand. 179, 5-8. doi: 10.1046/j.1365-201X.2003.01195.x

Eltzschig, H. K., Sitkovsky, M. V., and Robson, S. C. (2012). Purinergic signaling during inflammation. N. Engl. J. Med. 367, 2322-2333. doi: 10.1056/NEJMra1205750

Essenfelder, G. M., Bruzzone, R., Lamartine, J., Charollais, A., Blanchet-Bardon, C., Barbe, M. T., et al. (2004). Connexin30 mutations responsible for hidrotic ectodermal dysplasia cause abnormal hemichannel activity. Hum. Mol. Genet. 13, 1703-1714. doi: 10.1093/hmg/ddh191

Fang, X., Huang, T., Zhu, Y., Yan, Q., Chi, Y., Jiang, J. X., et al. (2011). Connexin43 hemichannels contribute to cadmium-induced oxidative stress and cell injury. Antioxid. Redox Signal. 14, 2427-2439. doi: 10.1089/ars.2010.3150

Figueroa, X. F., Lillo, M. A., Gaete, P. S., Riquelme, M. A., and Sáez, J. C. (2013). Diffusion of nitric oxide across cell membranes of the vascular wall requires specific connexin-based channels. Neuropharmacology 75, 471-478. doi: 10.1016/j. neuropharm.2013.02.022

Fiori, M. C., Figueroa, V., Zoghbi, M. E., Saéz, J. C., Reuss, L., and Altenberg, G. A. (2012). Permeation of calcium through purified connexin 26 hemichannels. J. Biol. Chem. 287, 40826-40834. doi: 10.1074/jbc.M112.383281

Froger, N., Orellana, J. A., Calvo, C. F., Amigou, E., Kozoriz, M. G., Naus, C. C., et al. (2010). Inhibition of cytokine-induced connexin43 hemichannel activity in astrocytes is neuroprotective. Mol. Cell. Neurosci. 45, 37-46. doi: 10.1016/j.mcn.2010.05.007

Gaston, B. M., Carver, J., Doctor, A., and Palmer, L. A. (2003). S-nitrosylation signaling in cell biology. Mol. Interv. 3, 253-263. doi: 10.1124/mi.3.5.253

Gerido, D. A., DeRosa, A. M., Richard, G., and White, T. W. (2007). Aberrant hemichannel properties of Cx26 mutations causing skin disease and deafness. Am. J. Physiol. Cell Physiol. 293, C337-C345. doi: 10.1152/ajpcell.00626.2006

Goldberg, G. S., Lampe, P. D., and Nicholson, B. J. (1999). Selective transfer of endogenous metabolites through gap junctions composed of different connexins. Nat. Cell Biol. 1, 457-459. doi: 10.1038/15693

Iglesias, R., Locovei, S., Roque, A., Alberto, A. P., Dahl, G., Spray, D. C., et al. (2008). P2X7 receptor-Pannexin1 complex: pharmacology and signaling. Am. J. Physiol. Cell Physiol. 295, C752-C760. doi: 10.1152/ajpcell.00228.2008

Ishiura, S. (1981). Calcium-dependent proteolysis in living cells. Life Sci. 29, 1079-1087. doi: 10.1016/0024-3205(81)90194-6

Johnstone, S. R., Billaud, M., Lohman, A. W., Taddeo, E. P., and Isakson, B. E. (2012). Posttranslational modifications in connexins and pannexins. J. Membr. Biol. 245, 319-332. doi: 10.1007/s00232-012-9453-3

Ju, Y., Zhang, W., Pei, Y., and Yang, G. (2013). H(2)S signaling in redox regulation of cellular functions. Can. J. Physiol. Pharmacol. 91, 8-14. doi: 10. 1139/cjpp-2012-0293 
Kam, Y., Kim, D. Y., Koo, S. K., and Joe, C. O. (1998). Transfer of second messengers through gap junction connexin 43 channels reconstituted in liposomes. Biochim. Biophys. Acta 1372, 384-388. doi: 10.1016/S0005-2736 (98)00075-3

Kamermans, M., Fahrenfort, I., Schultz, K., Janssen-Bienhold, U., Sjoerdsma, T., and Weiler, R. (2001) Hemichannel-mediated inhibition in the outer retina. Science 20292, 1178-1180. doi: 10.1126/science.1060101

Kar, R., Batra, N., Riquelme, M. A., and Jiang, J. X. (2012). Biological role of connexin intercellular channels and hemichannels. Arch. Biochem. Biophys. 524, 2-15. doi: 10.1016/ j.abb.2012.03.008

Kolluru, G. K., Shen, X., and Kevil, C. G. (2013). A tale of two gases: NO and H2S, foes or friends for life? Redox Biol. 1, 313-318. doi: 10.1016/j.redox.2013.05.001

Kronengold, J., Srinivas, M., and Verselis, V. K. (2012). The N-terminal half of the connexin protein contains the core elements of the pore and voltage gates. J. Membr. Biol. 245, 453-463. doi: 10.1007/s00232-012-9457-z

Lam, Y. W., Yuan, Y., Isaac, J., Babu, C. V., Meller, J., and Ho, S. M. (2010). Comprehensive identification and modified-site mapping of S-nitrosylated targets in prostate epithelial cells. PLoS ONE. 5:e9075. doi: 10.1371/journal.pone.0009075

Lampe, P. D., and Lau, A. F. (2000). Regulation of gap junctions by phosphorylation of connexins. Arch. Biochem. Biophys. 384, 205-215. doi: 10.1006/abbi. 2000.2131

Leclerc, P. C., Lanctot, P. M., Auger-Messier, M., Escher, E., Leduc, R., and Guillemette, G. (2006) S-nitrosylation of cysteine 289 of the AT1 receptor decreases its affinity for angiotensin II. Br. J. Pharmacol. 148, 306-313. doi: 10.1038/sj.bjp.0706725

Levit, N. A., Mese, G., Basaly, M. G., and White, T. W. (2012). Pathological hemichannels associated with human Cx26 mutations causing KeratitisIchthyosis-Deafness syndrome. Biochim. Biophys. Acta 1818, 2014-2019. doi: 10.1016/j.bbamem.2011.09.003

Liang, G. S., de Miguel, M., Gómez-Hernández, J. M., Glass, J. D., Scherer, S. S., Mintz, M., et al. (2005). Severe neuropathy with leaky connexin32 hemichannels. Ann. Neurol. 57, 749-754. doi: 10.1002/ana.20459

Locovei, S., Wang, J., and Dahl, G. (2006). Activation of pannexin 1 channels by ATP through P2Y receptors and by cytoplasmic calcium. FEBS Lett. 580, 239-244. doi: 10.1016/j.febslet.2005.12.004

Lohman, A. W., Weaver, J. L., Billaud, M., Sandilos, J. K., Griffiths, R., Straub, A. C., et al. (2012). S-nitrosylation inhibits pannexin 1 channel function. J. Biol. Chem. 287, 39602-39612. doi: 10.1074/jbc.M112.397976

Milks, L. C., Kumar, N. M., Houghten, R., Unwin, N., and Gilula, N. B. (1988) Topology of the 32-kd liver gap junction protein determined by site-directed antibody localizations. EMBO J. 7, 2967-2975.

Moreno, A. P. (2005). Connexin phosphorylation as a regulatory event linked to channel gating. Biochim. Biophys. Acta 1711, 164-171. doi: 10.1016/j.bbamem.2005.02.016

Morlé, L., Bozon, M., Alloisio, N., Latour, P., Vandenberghe, A., Plauchu, H., et al. (2000). A novel C202F mutation in the connexin 26 gene (GJB2) associated with autosomal dominant isolated hearing loss. Med. Genet. 37, 368-370. doi: 10.1136/jmg.37.5.368

Nakamura, T., Tu, S., Akhtar, M. W., Sunico, C. R., Okamoto, S., and Lipton, S. A. (2013). Aberrant protein s-nitrosylation in neurodegenerative diseases. Neuron 78, 596-614. doi: 10.1016/j.neuron.2013.05.005

Niessen, H., Harz, H., Bedner, P., Krämer, K., and Willecke, K. (2000). Selective permeability of different connexin channels to the second messenger inositol 1,4,5-trisphosphate. J. Cell Sci. 113, 1365-1372.

Orellana, J. A., Froger, N., Ezan, P., Jiang, J. X., Bennett, M. V., Naus, C. C., et al. (2011). ATP and glutamate released via astroglialconnexin 43 hemichannels mediate neuronal death through activation of pannexin 1 hemichannels. J. Neurochem. 118, 826-840. doi: 10.1111/j.1471-4159.2011.07210.x

Orellana, J. A., Avendaño, B. C., and Montero, T. D. (2013). Role of connexins and pannexins in ischemic stroke. Curr. Med. Chem. [Epub ahead of print].

Pelegrin, P., and Surprenant, A. (2006). Pannexin-1 mediates large pore formation and interleukin-1beta release by the ATP-gated P2X7 receptor. EMBO J. 25, 5071-5082. doi: 10.1038/sj.emboj.7601378

Penuela, S., Bhalla, R., Gong, X. Q., Cowan, K. N., Celetti, S. J., Cowan, B. J., et al. (2007). Pannexin 1 and pannexin 3 are glycoproteins that exhibit many distinct characteristics from the connexin family of gap junction proteins. J. Cell Sci. 120, 3772-3783. doi: 10.1242/jcs.009514
Penuela, S., Gehi, R., and Laird, D. W. (2013). The biochemistry and function of pannexin channels. Biochim. Biophys. Acta 1828, 15-22. doi: 10.1016/j.bbamem.2012.01.017

Peracchia, C. (2004). Chemical gating of gap junction channels; roles of calcium, pH and calmodulin. Biochim. Biophys. Acta. 1662, 61-80. doi: 10.1016/j.bbamem.2003.10.020

Ponsaerts, R., De Vuyst, E., Retamal, M., D’Hondt C., Vermeire, D., Wang, N., et al. (2010). Intramolecular loop/tail interactions are essential for connexin 43-hemichannel activity. FASEB J. 24, 4378-4395. doi: 10.1096/fj. 09-153007

Prochnow, N., Hoffmann, S., Dermietzel, R., and Zoidl, G. (2009). Replacement of a single cysteine in the fourth transmembrane region of zebrafish pannexin 1 alters hemichannel gating behavior. Exp. Brain Res. 199, 255-264. doi: 10.1007/s00221-009-1957-4

Rackauskas, M., Neverauskas, V., and Skeberdis, V. A.(2010). Diversity and properties of connexin gap junction channels. Medicina (Kaunas). 46, 1-12.

Ramachandran, S., Xie, L. H., John, S. A., Subramaniam, S., and Lal, R. (2007). A novel role for connexin hemichannel in oxidative stress and smoking-induced cell injury. PLoS ONE. 2:e712. doi: 10.1371/journal.pone.0000712

Rasmussen, H., Barrett, P., Smallwood, J., Bollag, W., and Isales, C. (1990). Calcium ion as intracellular messenger and cellular toxin. Environ. Health Perspect. 84, 17-25. doi: 10.1289/ehp.908417

Retamal, M. A., Cortés, C. J., Reuss, L., Bennett, M. V., and Sáez, J. C. (2006). Snitrosylation and permeation through connexin 43 hemichannels in astrocytes: induction by oxidant stress and reversal by reducing agents. Proc. Natl. Acad. Sci. U.S.A. 103, 4475-4480. doi: 10.1073/pnas.0511118103

Retamal, M. A., Froger, N., Palacios-Prado, N., Ezan, P., Sáez, P. J., Sáez, J. C., et al. (2007a). Cx43 hemichannels and gap junction channels in astrocytes are regulated oppositely by proinflammatory cytokines released from activated microglia.J. Neurosci. 27, 13781-13792. doi: 10.1523/JNEUROSCI.204207.2007

Retamal, M. A., Schalper, K. A., Shoji, K. F., Bennett, M. V., and Sáez, J. C. (2007b). Opening of connexin 43 hemichannels is increased by lowering intracellular redox potential. Proc. Natl. Acad. Sci. U.S.A. 104, 8322-8327. doi: 10.1073/pnas.0702456104

Retamal, M. A., Yin, S., Altenberg, G. A., and Reuss, L. (2009). Modulation of Cx46 hemichannels by nitric oxide. Am. J. Physiol. Cell Physiol. 296, C1356-C1363. doi: $10.1152 /$ ajpcell. 00054.2009.

Retamal, M. A., León-Paravic, C. G., Verdugo, C. A., Alcaino, C. A., and MoragaAmaro, R. (2011). Connexin in lens physiology and cataract formation. J. Clin. Exp. Ophthalmol. S1:001. doi: 10.4172/2155-9570.S1-001

Riteau, N., Gasse, P., Fauconnier, L., Gombault, A., Couegnat, M., Fick, L., et al. (2010). Extracellular ATP is a danger signal activating P2X7 receptor in lung inflammation and fibrosis. Am. J. Respir. Crit. Care Med. 182, 774-783. doi: 10.1164/rccm.201003-0359OC

Romanello, M., and D'Andrea P. (2001). Dual mechanism of intercellular communication in HOBIT osteoblastic cells: a role for gap-junctional hemichannels. J. Bone Miner. Res. 16, 1465-1476. doi: 10.1359/jbmr.2001.16. 8.1465

Sáez, J. C., Connor, J. A., Spray, D. C., and Bennett, M. V. (1989). Hepatocyte gap junctions are permeable to the second messenger, inositol 1,4,5-trisphosphate, and to calcium ions. Proc. Natl. Acad. Sci. U.S.A. 86, 2708-2712. doi: 10.1073/ pnas.86.8.2708

Sáez, J. C., Martínez, A. D., Brañes, M. C., and González, H. E. (1998). Regulation of gap junctions by protein phosphorylation. Braz. J. Med. Biol. Res. 31, 593-600.

Sáez, J. C., Schalper, K. A., Retamal, M. A., Orellana, J. A., Shoji, K. F., and Bennett, M. V. (2010). Cell membrane permeabilization via connexin hemichannels in living and dying cells. Exp. Cell Res. 316, 2377-2389. doi: 10.1016/j.yexcr.2010.05.026

Sánchez, H. A., Mese, G., Srinivas, M., White, T. W., and Verselis, V. K. (2010). Differentially altered $\mathrm{Ca}^{2+}$ regulation and $\mathrm{Ca}^{2+}$ permeability in Cx26 hemichannels formed by the A40V and G45E mutations that cause keratitis ichthyosis deafness syndrome. J. Gen. Physiol. 136, 47-62. doi: 10.1085/jgp.201010433

Schalper, K. A., Sánchez, H. A., Lee, S. C., Altenberg, G. A., Nathanson, M. H., and Sáez, J. C. (2010). Connexin 43 hemichannels mediate the $\mathrm{Ca}^{2+}$ influx induced by extracellular alkalinization. Am. J. Physiol. Cell Physiol. 299, C1504-C1515. doi: 10.1152/ajpcell.00015.2010 
Schmidt, H. H., Pollock, J. S., Nakane, M., Förstermann, U., and Murad, F. (1992). $\mathrm{Ca}^{2+} /$ calmodulin-regulated nitric oxide synthases. Cell Calcium 13, 427-434. doi: 10.1016/0143-4160(92)90055-W

Scemes, E., Suadicani, S. O., Dahl, G., and Spray, D. C. (2007). Connexin and pannexin mediated cell-cell communication. Neuron Glia Biol. 2007, 199-208. doi: 10.1017/S1740925X08000069

Seminario-Vidal, L., Kreda, S., Jones, L., O’Neal, W., Trejo, J., Boucher, R. C., et al. (2009). Thrombin promotes release of ATP from lung epithelial cells through coordinated activation of rho- and $\mathrm{Ca}^{2+}$ dependent signaling pathways. J. Biol. Chem. 284, 20638-20648. doi: 10.1074/jbc.M109.004762

Sillén, A., and Annerén, G., and Dahl, N. (1998). A novel mutation (C201R) in the transmembrane domain of connexin 32 in severe X-linked CharcotMarie-Tooth disease. Hum. Mutat. 1998 (Suppl. 1), S8-S9. doi: 10.1002/humu. 1380110104

Song, D., Liu, X., Liu, R., Yang, L., Zuo, J., and Liu, W. (2010). Connexin 43 hemichannel regulates $\mathrm{H} 9 \mathrm{c} 2$ cell proliferation by modulating intracellular ATP and $\left[\mathrm{Ca}^{2+}\right]$. Acta Biochim. Biophys. Sin. (Shanghai). 42, 472-482. doi: 10.1093/abbs/gmq047

Sosinsky, G. E., Boassa, D., Dermietzel, R., Duffy, H. S., Laird, D. W., MacVicar, B., et al. (2011). Pannexin channels are not gap junction hemichannels. Channels (Austin). 5, 193-197. doi: 10.4161/chan.5.3.15765

Stehberg, J., Moraga-Amaro, R., Salazar, C., Becerra, A., Echeverría, C., Orellana, J. A., et al. (2012). Release of gliotransmitters through astroglialconnexin 43 hemichannels is necessary for fear memory consolidation in the basolateral amygdala. FASEB J. 26, 3649-3657. doi: 10.1096/fj.11-198416

Stong, B. C., Chang, Q., Ahmad, S., and Lin, X. (2006). A novel mechanism for connexin 26 mutation linked deafness: cell death caused by leaky gap junction hemichannels. Laryngoscope 116, 2205-2210. doi: 10.1097/01.mlg.0000241944.77192.d2

Stout, C. E., Costantin, J. L., Naus, C. C., and Charles, A. C. (2002). Intercellular calcium signaling in astrocytes via ATP release through connexin hemichannels. J. Biol. Chem. 277, 10482-10488. doi: 10.1074/jbc.M109902200

Straub, A. C., Billaud, M., Johnstone, S. R., Best, A. K., Yemen, S., Dwyer, S. T., et al. (2011). Compartmentalized connexin 43s-nitrosylation/denitrosylation regulates heterocellular communication in the vessel wall. Arterioscler. Thromb. Vasc. Biol. 31, 399-407. doi: 10.1161/ ATVBAHA. 110.215939

Thompson, R. J., Zhou, N., and MacVicar, B. A. (2006). Ischemia opens neuronal gap junction hemichannels. Science 312, 924-927. doi: 10.1126/science.1126241

Tong, J. J., Minogue, P. J., Guo, W., Chen, T. L., Beyer, E. C., Berthoud, V. M., et al. (2011) Different consequences of cataract-associated mutations at adjacent positions in the first extracellular boundary of connexin50. Am. J. Physiol. Cell Physiol. 300, C1055-C1064. doi: 10.1152/ajpcell.00384.2010

Valiunas, V. (2013). Cyclic nucleotide permeability through unopposed connexin hemichannels. Front. Pharmacol. 4:75. doi: 10.3389/fphar.2013.00075
Verselis, V. K., and Srinivas, M. (2008). Divalent cations regulate connexin hemichannels by modulating intrinsic voltage-dependent gating. J. Gen. Physiol. 132, 315-327. doi: 10.1085/jgp.200810029

Vroman, R., Klaassen, L. J., and Kamermans, M. (2013). Ephaptic communication in the vertebrate retina. Front. Hum. Neurosci. 7:612. doi: 10.3389/fnhum.2013.00612

Wang, Y., Chen, C., Loake, G. J., and Chu, C. (2010). Nitric oxide: promoter or suppressor of programmed cell death? Protein Cell 1, 133-142. doi: 10.1007/s13238010-0018-x

Wang, J., and Dahl, G. (2010). SCAM analysis of Panxl suggests a peculiar pore structure. J. Gen. Physiol. 136, 515-527. doi: 10.1085/jgp.201010440

Weilinger, N. L., Tang, P. L., and Thompson, R. J. (2012). Anoxia-induced NMDA receptor activation opens pannexin channels via Src family kinases. J. Neurosci. 32, 12579-12588. doi: 10.1523/JNEUROSCI.1267-12.2012

Wilkinson, W. J., and Kemp, P. J. (2011). Carbon monoxide: an emerging regulator of ion channels. J. Physiol. 589, 3055-3062. doi: 10.1113/J Physiol. 2011.206706

Ye, Z. C., Wyeth, M. S., Baltan-Tekkok, S., and Ransom, B. R. (2003). Functional hemichannels in astrocytes: a novel mechanism of glutamate release. J. Neurosci. 23, 3588-3596.

Yeager, M., and Gilula, N. B.(1992). Membrane topology and quaternary structure of cardiac gap junction ion channels. J. Mol. Biol. 223, 929-948. doi: 10.1016/0022-2836(92)90253-G

Zhang, L., Deng, T., Sun, Y., Liu, K., Yang, Y., and Zheng, X. (2008). Role for nitric oxide in permeability of hippocampal neuronal hemichannels during oxygen glucose deprivation. J. Neurosci. Res. 86, 2281-2291. doi: 10.1002/ jnr.21675

Conflict of Interest Statement: The author declares that the research was conducted in the absence of any commercial or financial relationships that could be construed as a potential conflict of interest.

Received: 14 November 2013; accepted: 08 February 2014; published online: 25 February 2014.

Citation: Retamal MA (2014) Connexin and Pannexin hemichannels are regulated by redox potential. Front. Physiol. 5:80. doi: 10.3389/fphys.2014.00080

This article was submitted to Membrane Physiology and Membrane Biophysics, a section of the journal Frontiers in Physiology.

Copyright (C) 2014 Retamal. This is an open-access article distributed under the terms of the Creative Commons Attribution License (CC BY). The use, distribution or reproduction in other forums is permitted, provided the original author(s) or licensor are credited and that the original publication in this journal is cited, in accordance with accepted academic practice. No use, distribution or reproduction is permitted which does not comply with these terms. 